\title{
Micro-Neurosurgical System in the Deep Surgical Field
}

\author{
Daisuke Asai ${ }^{1}$, Surman Katopo ${ }^{1}$, Jumpei Arata ${ }^{1}$, \\ Shin'ichi Warisawa ${ }^{1}$, Mamoru Mitsuishi ${ }^{1}$, \\ Akio Morita $^{2}$, Shigeo Sora ${ }^{2}$, Takaaki Kirino ${ }^{2}$, and Ryo Mochizuki ${ }^{3}$ \\ 1 School of Engineering, The University of Tokyo \\ 7-3-1, Hongo, Bunkyo-ku, Tokyo 113-8656, Japan \\ \{daisuke, surman, jumpei, warisawa, mamoru\}@nml.t.u-tokyo.ac.jp \\ 2 School of Medicine, The University of Tokyo \\ \{amor-tky, sora-tky, tkirino-tky\}@umin.ac.jp \\ 3 NHK Engineering Services, Inc. \\ 1-10-11, Kinuta, Setagaya-ku, Tokyo 157-8540, Japan \\ r-mochi@cyborg.ne.jp
}

\begin{abstract}
In neurosurgery, surgeons have to perform precise manipulations with poor visibility due to the presence of blood or cerebrospinal fluid and it is particularly difficult to operate in the deep surgical field. The authors have developed a microsurgical system for neurosurgery in the deep surgical field that addresses these difficulties. The authors succeeded in suturing the carotid artery of a rat under a glass tube 120 [mm] in depth and $50[\mathrm{~mm}]$ in diameter. In this paper, the authors propose the concept of robotic-assisted micro-neurosurgery. The design and the system are presented. Furthermore, the performance of the system and in-vivo experiments on rats are also reported.
\end{abstract}

\section{Introduction}

Neurosurgery has progressed rapidly since surgical microscopes were introduced and widely used in the 1970s. The development of the microscope provided an environment where microsurgery could be routinely executed in an enlarged operation field, enabling surgeons to perform various kinds of operative techniques which had been impossible in conventional surgery safely and precisely. However, it is still a challenge to reduce the invasiveness of microsurgery while protecting normal brain tissue during surgery. Many systems to support operative techniques in neurosurgery [1] [2, including master-slave systems to support microsurgery 3] [4, have been developed. The master-slave systems designate the operation site as the master and the surgical site as the slave. Micro-manipulation is performed by scaling the master and the slave manipulator motions [5]. The HUMAN system is a master-slave microsurgery system for neurosurgery 6] [7. Its insertion probe is 10 [mm] in diameter and contains a stereoscopic endoscope and three micro-manipulators each of which is $3[\mathrm{~mm}]$ in diameter and has 3 D.O.F. However, the motion range is limited to a $10[\mathrm{~mm}]$ cube, which 


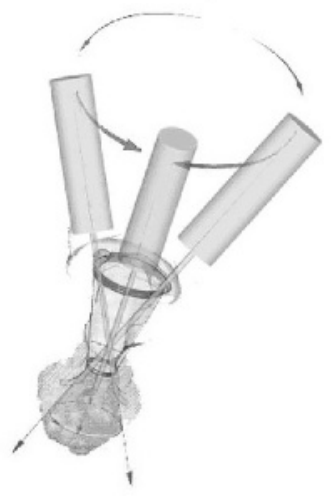

Fig. 1. Concept for the forceps

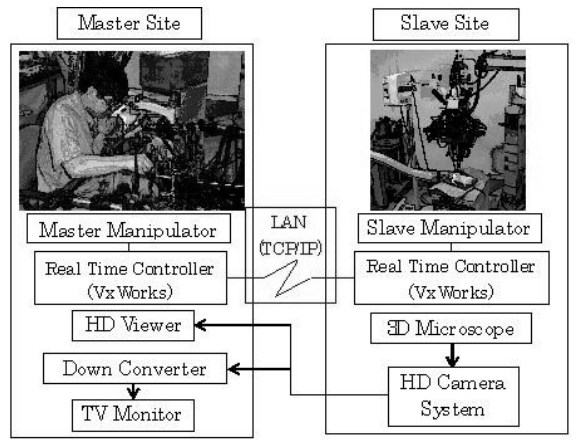

Fig. 2. Overview of the system

is not enough to complete a surgical operation, such as suturing and removing tumors. Da Vinci [8] and ZEUS 9] are already available commercially and have been used in many clinical applications. However, they are not appropriate for minimally invasive neurosurgery because they were developed to support laparoscopic or heart surgery and their insertion probes are more than $10[\mathrm{~mm}]$ in diameter. To solve the problems, the authors developed a microsurgical system for neurosurgery to assist micro-manipulation in the deep surgical field.

\section{Design of a Neurosurgical System}

\subsection{Concept}

To decrease the invasiveness of neurosurgery, it is necessary to provide a sufficiently enlarged image of the target and precise maneuverability to the surgeon. The former requirement is fulfilled with by higher-powered surgical microscope. However, the latter requirement depends mostly on surgeon's personal skill, which is acquired by specialized training and refined through experience and continual application. Therefore, the robotic surgical assistance system is expected to be used in difficult operative procedures. The requirements and necessary conditions for a robotic surgical assistance system for neurosurgery are as follows:

- Precise maneuverability sufficient to suture a micro blood vessel,

- Insertion through a small craniotomy hall to decrease invasiveness,

- Sufficient maneuverability in the deep surgical field,

- To provide the expanded surgical field to a surgeon,

- To assure the mechanical safety of patients, and

- To adapt to the current neurosurgery environment. 


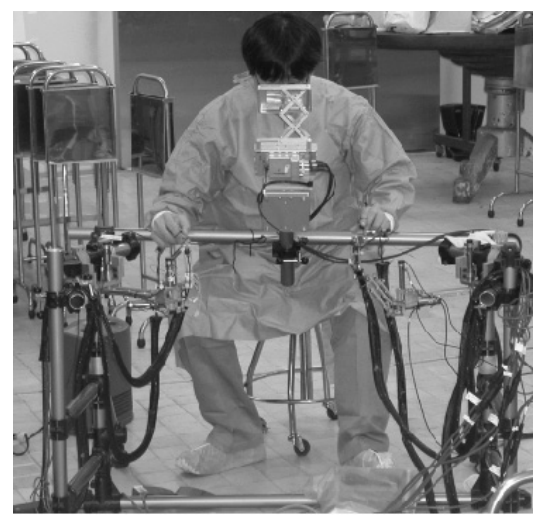

Fig. 3. Master manipulator

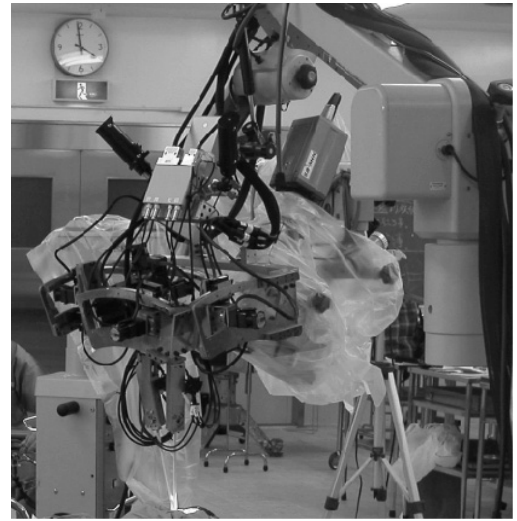

Fig. 4. Slave manipulator

\subsection{System Requirement}

Based on requirements and necessary conditions mentioned above, the authors set the following specifications for the microsurgical system:

- Positioning accuracy of better than $50[\mu \mathrm{m}]$,

- Diameter of the forceps less than $5[\mathrm{~mm}]$,

- The forceps have 1 bending D.O.F. and a range of motion from -90 [deg] to +90 [deg],

- There is a fixed point at the insertion part of the forceps,

- $100[\mathrm{~mm}]$ translational motion range in the inserting direction,

- The forceps can be changed easily, and

- The forceps can be sterilized and irrigated easily.

\section{Implementation of the System}

\subsection{Overview of the System}

Fig 2 shows a block diagram of the microsurgical system for neurosurgery in the deep surgical field. The system consists of three parts: the master manipulator which the surgeon operates, the slave manipulator which performs actual surgical operations on the patient, and the 3-dimensional surgical microscope system.

\subsection{Master Manipulator}

The master manipulator is an interface device that a surgeon operates. The surgeon can control the slave manipulator by applying motion to the device. Fig 3 shows an overview of the master manipulator. It consists of left and right arms. Each arm has 3 translational D.O.F., 3 rotational D.O.F., and 1 grasping D.O.F. In addition, it has 3 foot switches to assist operation. 


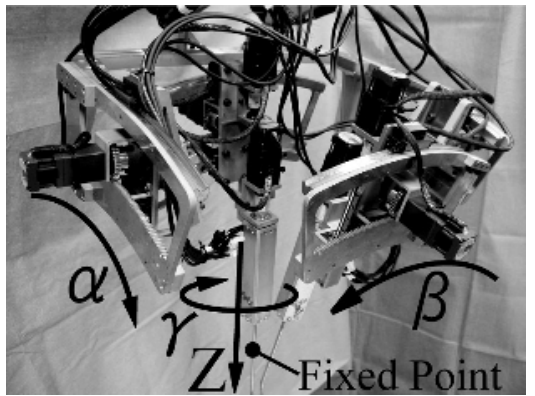

Fig. 5. Mechanism of the slave arm

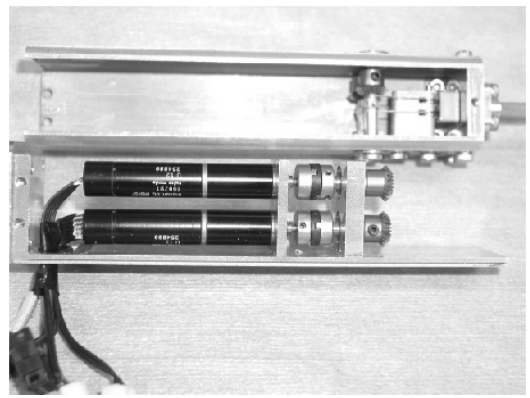

Fig. 6. Detachable actuators for the forceps

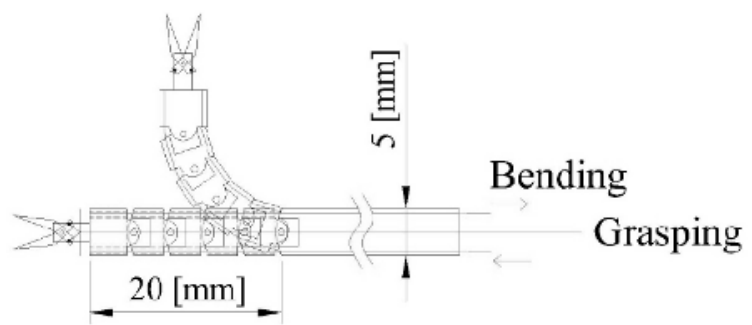

Fig. 7. Mechanism of the forceps

\subsection{Slave Manipulator}

The slave manipulator performs a surgical pocedure on a patient according to the commands from the master manipulator. Fig 4 shows an overview of the slave manipulator. It consists of three parts: the arm part which holds and moves surgical tools, the forceps part which is inserted in the brain, and the base part which determines the position of the arm.

1. Arm part

The arm part consists of two arms (left/right) to hold surgical tools (Fig 5 . Each arm has 3 rotational D.O.F. $(\alpha, \beta, \gamma)$, and 1 translational D.O.F. along the insertion axis $(Z)$. Radius guides were adopted for the rotational motion around $\alpha$ and $\beta$-axis. The axes intersect at the mechanical fixed point on the insertion axis. Safe, minimally invasive surgery is achieved by setting the point at the narrowest part of the pathway to the affected area, where the maximum motion limitation is required. The motion ranges of $\alpha, \beta, \gamma$ and $Z$-axis are from -15 to +15 [deg], from -15 to +15 [deg], from -270 to $+270[\mathrm{deg}]$, and from 0 to 90 [mm], respectively. Each axis is actuated by a stepping motor.

2. Forceps part

The micro-active forceps with a diameter of $5[\mathrm{~mm}]$ is detachable from the arm part. It has 1 bending D.O.F. and 1 grasping D.O.F. The length of the 

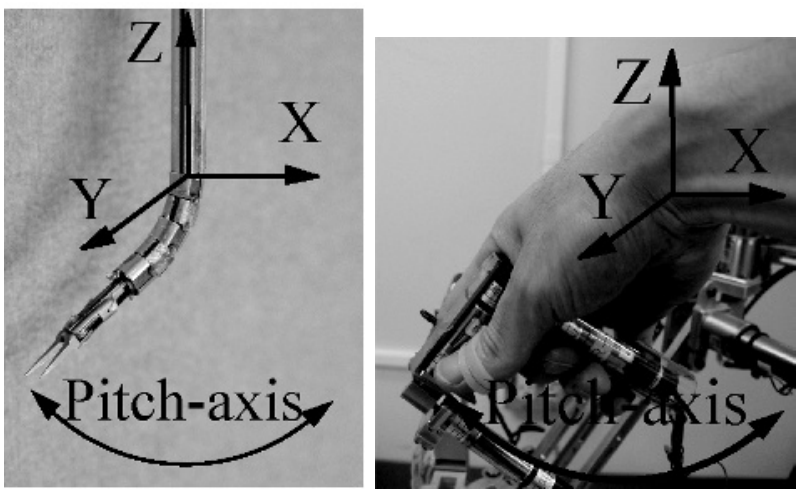

Fig. 8. Correspondence of D.O.F. between the master and the slave manipulator

bending part is $20[\mathrm{~mm}]$, the bending motion range is from -90 [deg] to +90 [deg]. The forceps has enough motion range to suture or to remove a tumor in the deep surgical field. Stainless wires transmit driving forces to bend and to grasp at the head of the forceps (Fig (7). An easily detachable mechanism between the insertion part and the motor of the forceps enables sterilization and irrigation of the insertion part (Fig 6] $)$. Each motion is actuated by a small DC motor.

3. Base part

The base part has 6 passive D.O.F. It determines the position of the arm part and the forceps part before the operation.

\subsection{Visual System}

To achieve micro-neurosurgery, an enlarged surgical field has to be provided to the surgeon who operates the master manipulator. In addition, good depth peception is crucial to successful operation in the deep surgical field. In the developed system, a high definition surgical view, which is obtained by the surgical microscope and a high definition camera for 3-dimensional display at the slave site, is presented on the 3 -dimensional display system at the master site. Using the developed system, the surgeon can operate more safely and precisely.

\subsection{Coordination Between Master and Slave Manipulator}

In an asymmetrical master-slave manipulator system, hand-eye coordination, which means how the slave manipulator in the display is to be moved according to the motion of the master manipulator by the surgeon, is very important. It is required for the system that the information obtained from the visual display and the operation sensation at the surgeon's hand should correspond. Therefore, a coordinate system transformation is performed between the master and the 


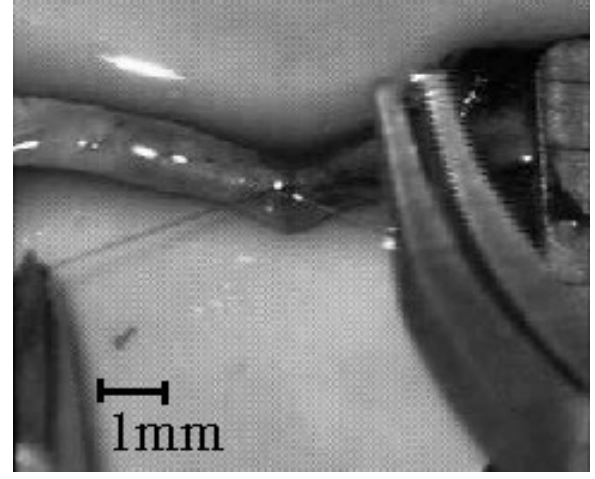

(a) Suturing a carotid artery

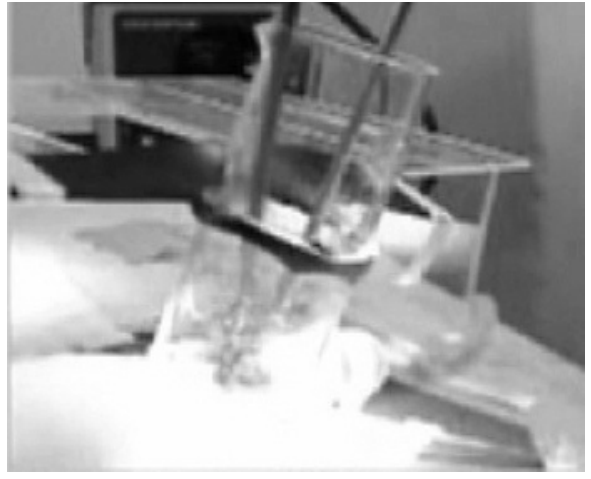

(b) A rat under a glass tube

Fig. 9. In-vivo experiment on a rat

slave manipulators shown in 8 . The bending motion at the head of the slave manipulator corresponds to a rotation around the surgeon's wrist. It enables the surgeon to operate the forceps as if he/she held its tip directly. It also reduces the force sensation felt by the surgeon.

\section{In Vivo Experiment by a Surgeon}

\subsection{Method}

To verify the effectiveness of the system for the exposure, dissection and suturing of a micro blood vessel in the deep surgical field, experiments were performed using rats (Fig 9). Exposure, dissection, and complete anastomosis of a common carotid artery were conducted on 10-week old male Wister rats by setting a glass tube (Fig 9 (b)), whose depth and the diameter are $90[\mathrm{~mm}]$ and $40[\mathrm{~mm}]$, respectively, to simulate an actual minimally invasive neurosurgery on a human brain and to limit the motion range of the forceps. A carotid artery was intentionally cutted off in advance and was sutured 10 to 13 times using a 10-0 needle and suture.

\subsection{Result and Discussion}

Exposure, dissection and complete anastomosis of the common carotid artery were successfully performed. The blood flow was confirmed without any leaks after suturing. During the suturing, at first the needle was grasped using the right forceps (needle handling) and then was stuck into the arterial wall (needle placement). It was pulled out by the left forceps, and was passed through the other arterial wall (arterial wall passing). Finally, the suture was knotted. The sum of the every operation time is shown in Fig.10 The average time to complete 


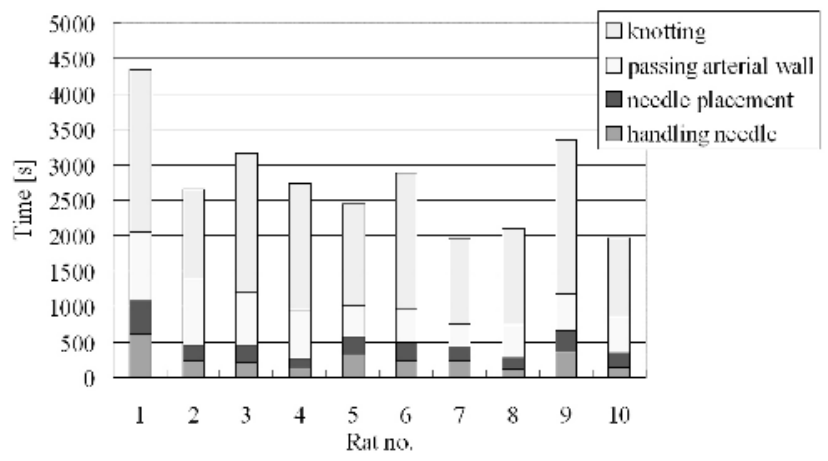

Fig. 10. Operation time
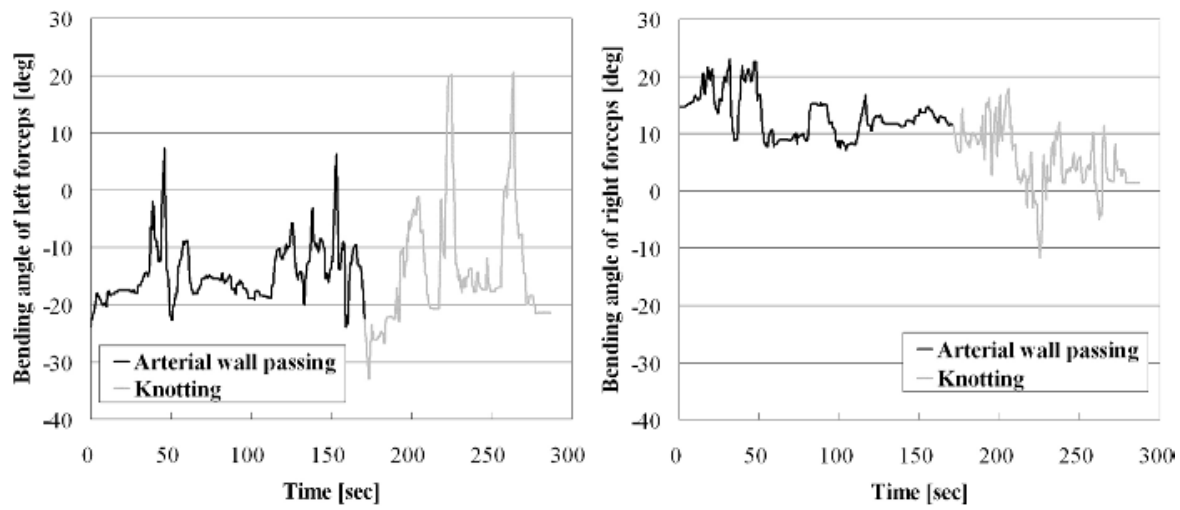

Fig. 11. The bending motions of right and left forceps in the in vivo experiment

anastomosis is 42.4 [min.]. Knotting required the longest and the most varied time. The target time is 20 [min.]. Fig[11 shows the bending angle of the left and the right forceps during each task. From the figure, it was determined that the suturing operation was performed by the cooperation of the left and the right forceps. Therefore, the effectiveness of the bending function was proved. Furthermore, blood flow could be confirmed after suturing 10 to 13 times. This demonstrates that the needle was inserted into the intended position around the incision. The experimental results show that the developed system has enough capability for micro-manipulation in deep and narrow spaces.

\section{Conclusion}

The authors have developed a microsurgical system for neurosurgery in the deep surgical field. The slave manipulator has two forceps, each of which has 1 D.O.F. in bending and 1 D.O.F. in grasping at its head. The length and the diameter of the insertion part are $190[\mathrm{~mm}]$ and $5[\mathrm{~mm}]$, respectively. The developed system 
provides an environment to suture and remove a tumor in the deep surgical field of the brain. The mechanical fixed point of the insertion probe was located at the narrowest section of the probe. It enables minimally invasive surgery to be performed more safely. To evaluate the effectiveness of the system, the authors have conducted a suturing operation on the common carotid arteries of 10 rats in a glass tube whose depth and diameter are 120 [mm] and 50 [mm], respectively. Blood flow was successfully confirmed after suturing. Further improvements of the system toward clinical application continue.

\section{References}

1. A. Perneczky and G. Fries, "Endoscope-assisted Brain Surgery: Part 1-Evolution, Basic Concept, and Current Technique," Neurosurgery, vol.42, no.2, pp.219-224, 1998.

2. B. Davis, S. Starkie, et al., "Neurobot: a special-purpose robot for Neurosurgery," Proc. of 2000 IEEE International Conference on Robotics and Automation, pp.41034108, 2000.

3. P. Le Roux, H. Das, et al., "Robotic-assisted Microsurgery: A Feasibility Study in the Rat," Neurosurgery, vol.48, no.3, pp.584-589, 2001.

4. M. Mitsuishi, Y. Iizuka, et al., "Remote Operation of a Micro-Surgical System," Proc. of IEEE 1998 International Conference on Robotics and Automation, pp.10131019, 1998.

5. S. E. Salcudean, et al., "Performance Measurement in Scaled Teleoperation for Microsurgery," CVRMed-MRCAS'97, pp.789-798, 1997.

6. N. Miyata, E. Kobayashi, et al., "Micro-grasping Forceps Manipulator for MRGuided Neurosurgery," Proc. Medical Image Computing and Computer-assisted Intervension 2002, vol.2488, pp.107-113, 2002.

7. K. Hongo, S. Kobayashi, Y. Kakizawa, et al., "Neurobot:Telecontrolled Micromanipulator System for Minimally Invasive Microneurosurgery-Preliminary Results," Neurosurgery, vol.51, no.4, pp.985-988, 2002.

8. G. Guthart and JK. Salisbury, "The Intuitive Telesurgery System: Overview and Application," Proc. of 2000 IEEE International Conference on Robotics and Automation, pp.618-621, 2000.

9. http://computermotion.com 